\title{
Performance Analysis of MIMO-STBC using Hamming Channel Codes for different modulation techniques
}

\author{
Khushboo Bisht ${ }^{1}$, Parminder Singh ${ }^{2}$,Dr.Sanjay Sharma ${ }^{3}$ \\ ${ }^{I}$ (Mtech Student, ECE Department, DIET, Kharar, Mohali) \\ ${ }^{2}$ (AP,ECE Department,Diet,Kharar,Mohali) \\ ${ }^{3}$ (H.O.D., Thapar University, Patiala)
}

\begin{abstract}
MIMO Diversity is a technique to mitigate the effect of fading and enhances the signal strength. $S T B C$ is used in MIMO systems to improve the performance by maximizing the diversity gain. In this paper, performance of MIMO-STBC for different modulatin techniques such as BPSK, QPSK, 8-PSK and 16-PSK using Hamming Coding is analysed on the basis of BER and SNR. A comparative study and performance analysis is done for SISO, SIMO, MISO and MIMO systems for BPSK modulation technique.
\end{abstract}

Keywords: Multiple- input Multiple-output (MIMO), Space Time Block Code(STBC), Alamouti, Bit Error Rate(BER),Hamming Coding .

\section{Introduction}

Wireless systems are rapidly developing to provide high speed data transfer but the major problem is data rate and range. To support these services large capacity channels are required. In wireless communication, the signal from transmitter is reached form various paths due to reflection, refraction, diffraction etc. Each path experiences different time delay, phase shift and attenuation which results in fluctuation in signal strength. This is known as multipath fading. As a result of fading multiple copies of transmitted signal is received at the receiver which degrades the system performance [1]. The basic idea is that if different copies of same signal are available then there is a high probability that at least one of them is of good quality [2].To improve the performance in fading channels diversity technique is used. We transmit different copies of signal through different channels instead of transmitting them over one channel. Diversity can be obtained by using multiple antennas both at transmitter and receiver end. This system is called multiple-input multiple-output (MIMO). These systems got higher consideration in Alamouti STBC where data is coded through space and time [3]. MIMO is based on both transmit and receive diversity. The overall effect MIMO systems can be summarized in terms of reduction of bit error rate and increase in system capacity [4]. This paper describes a wireless transmission using the concept of MIMO systems. MIMO using Space Time Block Coding (STBC) has been used with different modulation techniques such as BPSK, QPSK, 8-PSK and 16-PSK using hamming coding. This technique is capable of providing better data rates and significant reduction in bit error rate.

\section{Space Time Block Code}

Space time block code is presented by the Alamouti [5]. This scheme provides transmit and receive diversity to the MIMO systems. A simplified block diagram using Alamouti is described in fig.1. This scheme is defined by following three functions:

1. Encoding and deciding transmission sequence information symbols at the transmitter.

2. Combining signals with noise at the receiver.

3. Maximum likelihood detection. 


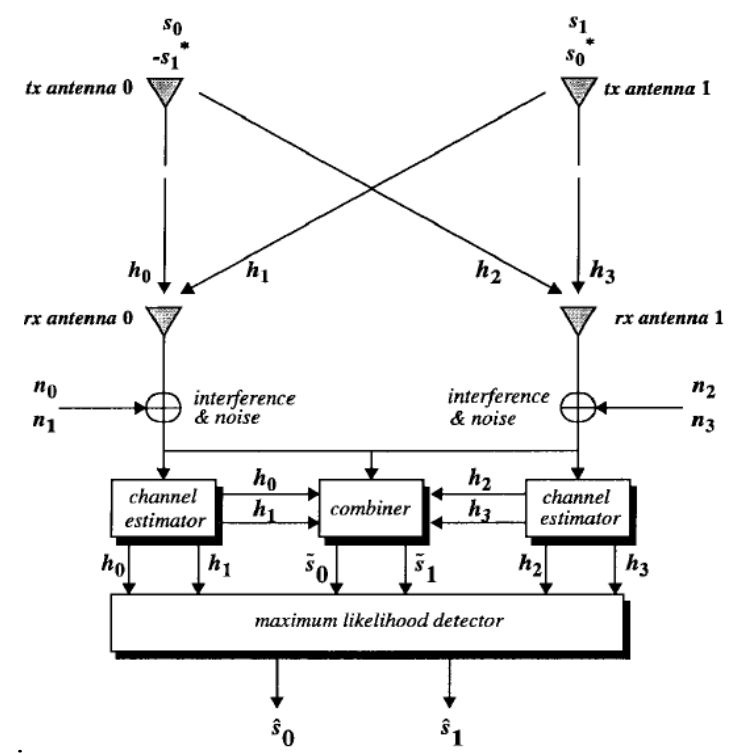

Fig.1. Simplified Alamouti space time coding (STC) for 2x2 MIMO systems [5].

At a given time period, two signals are simultaneously transmitted from two antennas. At time t, antenna 0 will transmit the signal $\mathrm{S}_{0}$ and antenna 1 will transmit the signal $\mathrm{S}_{1}$. At time $(\mathrm{t}+\mathrm{T})$, antenna 0 will transmit the signal $\left(-\mathrm{S}_{1}{ }^{*}\right)$ and antenna 1 will transmit the signal $\mathrm{S}_{0}{ }^{*}$ where $*$ is complex conjugate operation. The sequence is shown below:

Table 1. Encoding and transmission sequence for Alamouti STBC scheme [5]

\begin{tabular}{|c|c|c|}
\hline Time Slots & Antenna 0 & Antenna \\
\hline time $\mathrm{t}$ & $\mathrm{S}_{0}{ }^{*}$ & $\mathrm{~S}_{1}$ \\
\hline time $(\mathrm{t}+\mathrm{T})$ & $\left(-\mathrm{S}_{1}{ }^{*}\right)$ & $\mathrm{S}_{0}{ }^{*}$ \\
\hline
\end{tabular}

The received vectors can be expressed as,

$$
\begin{aligned}
& \mathrm{r}_{0}=\mathrm{h}_{0} \mathrm{~S}_{0}+\mathrm{h}_{1} \mathrm{~S}_{1}+\mathrm{n}_{0} \\
& \mathrm{r}_{1}=\mathrm{h}_{0}\left(-\mathrm{S}_{1}{ }^{*}\right)+\mathrm{h}_{1} \mathrm{~S}_{0}{ }^{*}+\mathrm{n}_{1} \\
& \mathrm{r}_{2}=\mathrm{h}_{2} \mathrm{~S}_{0}+\mathrm{h}_{3} \mathrm{~S}_{1}+\mathrm{n}_{2}
\end{aligned}
$$

$\mathrm{r}_{3}=\mathrm{h}_{2}\left(-\mathrm{S}_{1}^{*}\right)+\mathrm{h}_{3} \mathrm{~S}_{0}{ }^{*}+\mathrm{n}_{3}$

where $\mathrm{n}_{0}, \mathrm{n}_{1}, \mathrm{n}_{2}, \mathrm{n}_{3}$ are thermal noise and interference, $\mathrm{r}_{0}, \mathrm{r}_{1}, \mathrm{r}_{2}, \mathrm{r}_{3}$ denote the received vectors and $\mathrm{S}_{0}$ and $\mathrm{S}_{1}$ are modulated symbols.

The combiner will combine the two signals and then are sent to the maximum likelihood detector and expressed as,

$$
\begin{aligned}
& \tilde{S}_{0}=\mathrm{h}_{0}{ }^{*} \mathrm{r}_{0}+\mathrm{h}_{1} \mathrm{r}_{1}{ }^{*}+\mathrm{h}_{2}{ }^{*} \mathrm{r}_{2}+\mathrm{h}_{3} \mathrm{r}_{3}{ }^{*} \\
& \tilde{S}_{1}=\mathrm{h}_{1}{ }^{*} \mathrm{r}_{0}-\mathrm{h}_{0} \mathrm{r}_{1}{ }^{*}+\mathrm{h}_{3}{ }^{*} \mathrm{r}_{2}-\mathrm{h}_{2} \mathrm{r}_{3}{ }^{*}
\end{aligned}
$$

\section{System Model}

The system model is shown in fig.2. At first the data bits are transmitted through Rayleigh Fading channel. The channel encoder adds the redundant bits to the information pattern for error detection and correction. These data streams are modulated using M-PSK techniques. The signal is passed through Space Time Block Coder (STBC). Now all the modulated streams travel through the channel and then first decoded by STBC decoder and demodulated by M-PSK demodulator and then channel demodulator to get the received signal. 


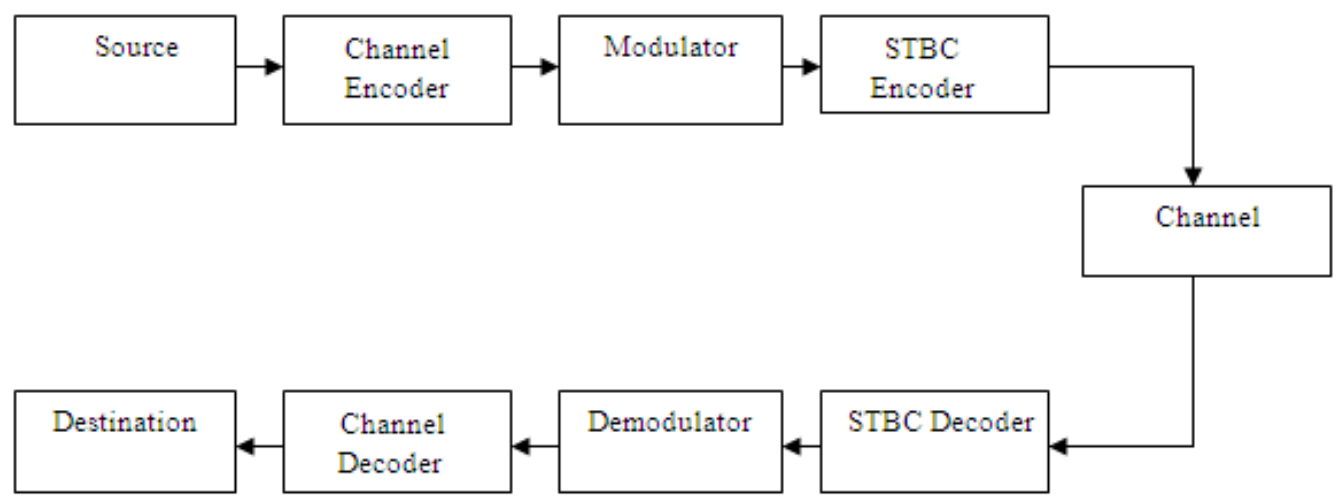

Fig.2. System Model [1]

\subsection{Transmitter} next stage.

The data is generated from random source, consists of series of zeroes and ones. The data is passed to

\subsubsection{CHANNEL ENCODER}

The Channel Encoder adds the redundant bits to the data bits for error correction and detection. In this model, hamming coding is used as a channel encoding technique. Hamming introduced the $(7,4)$ code. It encodes 4 data bits into 7 bits by adding three parity bits. Hamming $(7,4)$ can detect and correct single-bit errors.

\subsubsection{MODULATION}

The incoming data streams are modulated by using M-PSK techniques. There are four modulation techniques that are BPSK, QPSK, 8-PSK, and 16-PSK.

\subsubsection{STBC ENCODER}

Space Time Block Coding (STBC) based on Alamouti scheme based on both transmit and receive diversity [5]. $S_{0}$ and $S_{1}$ are the actual transmitted signal. The signal is passed through Rayleigh flat fading channel.

\subsubsection{STBC DECODER}

In the STBC decoder received signals are combined by maximal ratio combining and detected by maximum likelihood detection.

\subsubsection{DEMODULATOR}

Demodulator converts the modulated waveform created at the receiver to their original bits.

\subsubsection{CHANNEL DECODER}

At the receiver, channel decoding is done using syndrome decoding.

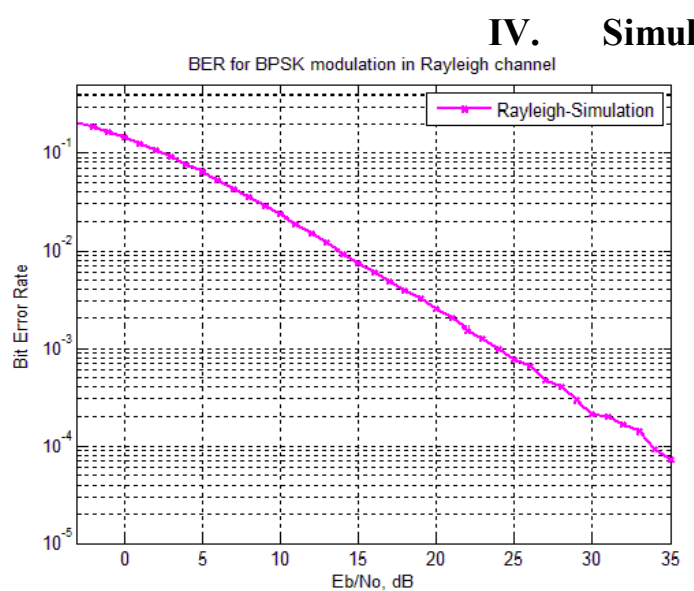

Fig.3 BER Plot of STBC for SISO system using BPSK

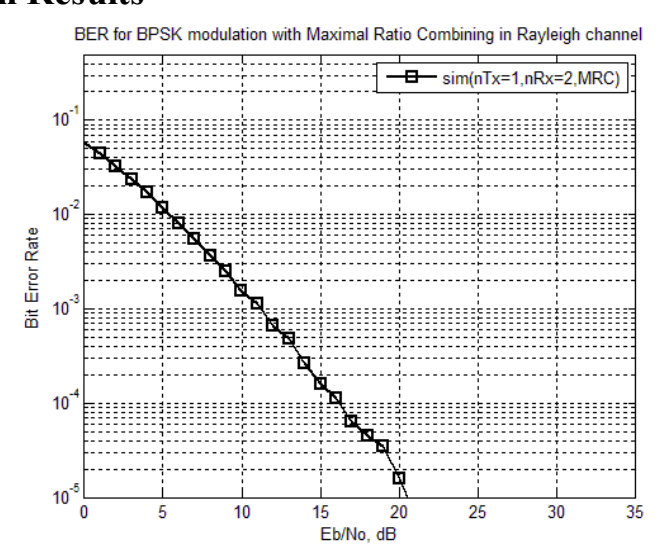

Fig.4 BER Plot of STBC for SIMO system using BPSK 


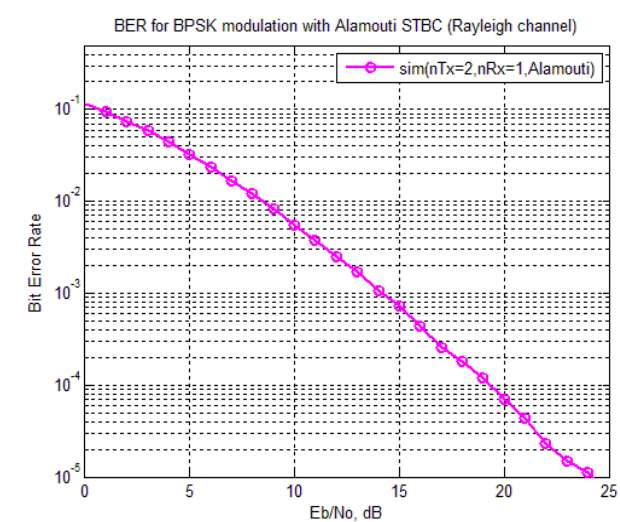

Fig.5 BER Plot of STBC for MISO system using BPSK

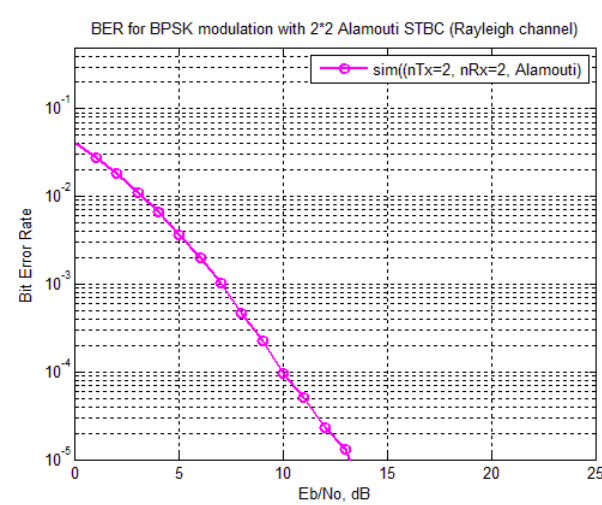

Fig.6 BER Plot of STBC for MIMO system using BPSK without channel coding

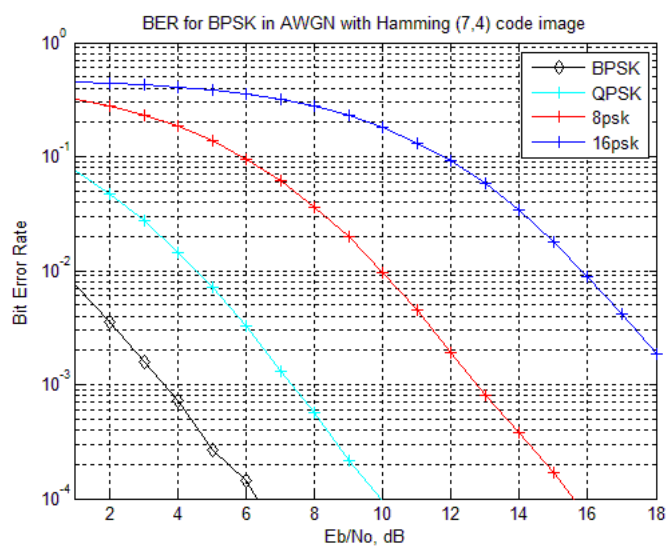

Fig.7 BER Plot of MIMO-STBC for BPSK, QPSK, 8-PSK, 16-PSK using Hamming Coding

In the simulation, the channel is assumed to be Rayleigh flat fading channel. The transmission employs different modulation techniques such as BPSK, QPSK, 8-PSK and 16-PSK. Maximum likelihood detection is done at the receiver for detection of signal. BPSK is more power efficient and needs less bandwidth. BPSK has less BER than the other techniques.

Table 2. SNR required at BER $5 * 10^{-2}$ for M-PSK for $2 * 2$ Alamouti Scheme with Hamming Coding

\begin{tabular}{|c|c|c|}
\hline Sr. No. & $\begin{array}{c}\text { Modulation } \\
\text { Scheme }\end{array}$ & SNR(db) \\
\hline 1 & BPSK & 1.2 \\
\hline 2 & QPSK & 5.3 \\
\hline 3 & 8-PSK & 10.8 \\
\hline 4 & 16-PSK & 16.5 \\
\hline
\end{tabular}

\section{Conclusion}

Space Time Block Codes with low order modulation always give low bit error rate as compared with Space Time Block Code with high order modulation. The result shows that Bit Error Rate (BER) of STBC with 16-PSK modulation is less for high SNR and BER with BPSK is less for low SNR. Thus STBC with BPSK is more power efficient and require less bandwidth.

\section{References}

[1]. Roshan Kumar, "Performance Analysis of STBC with channel coding", Thapar University, Patiala ,Punjab,2010.

[2]. Hafeth Hourani, "An overview of diversity techniques in wireless communication system", Helsinki University of Technology communication lab, 2004-05.

[3]. Bhawna Ahlawat and R.K.Reddy, "Channel Estimation and Performance Evaluation of STBC with channel coding”, International Journal of Engineering Research and Technology (IJERT), vol.1, issue 3, 2012.

[4]. Shubhangi Chaudhary and A.J.Patil, "Performance Analysis of MIMO STBC with different modulation techniques", ICTACT Journal on communication and technology, vol.03, issue 01, 2012.

[5]. S.M.Alamouti, "A simple transmitter diversity technique for wireless communications", IEEE Journal on selected areas communication, vol.16, No.8, pp.1451-1458,1998. 